\title{
Chaotic properties of a fully developed model turbulence
}

\author{
M. Yamada and Y. Saiki \\ Research Institute for Mathematical Sciences, Kyoto Univ., Kyoto, Japan
}

Received: 2 July 2007 - Revised: 4 September 2007 - Accepted: 11 September 2007 - Published: 25 September 2007

\begin{abstract}
Shell models of turbulence have been employed as toy models which, in their chaotic states, show statistical properties similar to real fluid turbulence, including Kolmogorov energy spectrum and intermittency. These models are interesting because, at the present stage, it is still quite difficult or almost impossible to study relations between those traditional statistical properties and the structure of the chaos underlying the real fluid turbulence because of huge dimension of the chaotic attractor. In this paper we will give a brief review on the chaotic properties of a shell model (GOY model), with emphasis on its Lyapunov spectrum and unstable periodic orbits, in relation to the Kolmogorov scaling law of the turbulence.
\end{abstract}

\section{Introduction}

The three-dimensional Navier-Stokes turbulence has long been a subject of research, where very unstable fluid motion is observed together with its robust statistical properties including the Kolmogorov energy spectrum and the intermittency. Several theories have been proposed to explain the statistical properties of turbulence, where attention was focused into the Kolmogorov spectrum itself in the early stage of turbulence study while higher-order correlations of velocity later attracted researchers' attention in relation to the intermittency, i.e., small deviations from the Kolmogorov scaling law observed in experiments.

From 1970s, on the other hand, it has been recognized that seemingly stochastic complex motions can be generated even in a deterministic dynamical system. The origin of the complex motion is irregular attracting sets in a phase space, which are called strange or chaotic attractors. The chaotic attractors in low-dimensional systems have been in-

Correspondence to: $\mathrm{M}$. Yamada

(yamada@kurims.kyoto-u.ac.jp) tensively investigated, and some aspects of the chaotic attractors have been studied in detail both analytically and numerically. However, most of the theoretical and numerical techniques employed in these studies cannot be applied to high-dimensional chaotic attractors because their procedures become rapidly complicated as the dimension of the attractor increases. At the present stage, therefore, it is still quite difficult to study the structure of a chaotic attractor of which the dimension exceeds some tens.

Along the line of chaos research, the Navier-Stokes turbulence has come to be considered as a typical and important dynamical phenomenon which is due to a chaotic attractor with, however, a huge dimension estimated to be more than at least some millions by using Kolmogorov scaling argument (Kida et al., 1989). Therefore, although it is now widely accepted that the Navier-Stokes turbulence is an example of chaos phenomena, and high-performance computers have become available recently, the computing power is still far from sufficient and properties of the underlying huge dimensional chaotic attractor are not well-understood yet, and especially we do not have an idea of what chaos properties correspond to the well-known robust statistical properties of fluid turbulence as the Kolmogorov scaling and the intermittency.

The present status of numerical calculation may allow two different ways of approach; one is to study only a few parameters of the full Navier-Stokes turbulence, and the other is to deal with a model turbulence of a small dimension to study a set of chaos parameters in detail. Shell models or cascade models of turbulence, one of which we discuss in this paper, belong to the latter approach (Bohr et al., 1998; Biferale, 2003). The shell model is a toy model of turbulence, which has a chaotic attractor of a low dimension usually less than some tens, but shares similar statistical properties to real turbulence. The shell model is not intended to approximate the real turbulence, and we do not try to justify the model equation by, for example, an asymptotic analysis.

Published by Copernicus Publications on behalf of the European Geosciences Union and the American Geophysical Union. 
The model equation is simply given by imitating the NavierStokes equation in Fourier space, and therefore is not unique in any sense. Our aim of the shell model research in this paper is to find a possible connection between the chaos properties and the turbulence statistics, although the connection, even if any, remains only a candidate at best for what is expected to take place in real turbulence.

In this paper we discuss the type of the shell model, which is sometimes called GOY (Gledzer-Ohkitani-Yamada) shell model. However, we here remark that this choice does not mean that the shell model is unique in some sense, but only that some amount of data has been accumulated with respect to its attractor and statistical properties.

We focus our attention first on Lyapunov spectrum of the turbulence. Lyapunov spectrum is known to be one of the most fundamental quantity describing the dynamical properties of chaotic motion. Lyapunov spectrum characterizes the instability of the chaotic orbit in phase space, and also is known to give, for example, an upper bound of the Hausdorff dimension of the chaotic attractor. However, Lyapunov spectrum consists of as many exponents as the system dimension, and is actually impossible to obtain in a huge system like the Navier-Stokes turbulence, except for the first few Lyapunov exponents. It is still unknown how the Lyapunov spectrum is in real fluid turbulence, and how it is related to the classical properties of the turbulence. In this paper we show that Lyapunov spectrum of the shell model can be obtained from the traditional Kolmogorov scaling law by taking into account the localization of Lyapunov vectors in phase space.

We are also interested in the scaling exponents of structure functions of velocity. The scaling exponents are one of the most fundamental quantities characterizing the statistical properties of a turbulent velocity field, and have been studied in most detail in turbulence research. The scaling exponent of the lowest order structure function, which is directly related to the energy spectrum, is known to agree well with the Kolmogorov's scaling theory (K41) (Kolmogorov, 1941), but the higher order structure functions are now known to have a deviation from the K41 theory, which is often called intermittency. Many phenomenological models of intermittency have been proposed so far to account for the qualitative or quantitative behavior of the scaling exponents (Kolmogorov, 1962; Parisi and Frisch, 1985; Meneveau and Sreenivasan, 1987; She and Leveque, 1994). However, no dynamical origin of the intermittency has yet been identified in view of dynamical system theory. In this paper we show that in the shell model turbulence, there are three kinds of unstable periodic solutions; the first one is the fixed point solution, the second one has a simple time-dependence with K41 scaling property with no intermittency, and the third one has a rather complicated orbit corresponding to the intermittency.

In the next section we briefly summarize the basic properties of the shell model. Lyapunov spectrum of the shell model is discussed in Sect. 3, and the analysis of unstable periodic orbits (UPOs) is described in Sect. 4. Conclusion is given in Sect. 5.

\section{A Shell model}

The energy cascade in fully developed turbulence has been modeled in several ways by low-dimensional systems of ordinary equations, in which dependent variables stand for dynamical variables associated with wavenumber band. These models, which are called cascade models or shell models, have been devised in the hope that they give an insight of the energy cascade process or more specifically the intermittency observed in the homogeneous isotropic turbulence. An early example of these models may be given by a cascade model proposed and studied by Desnyansky and Novikov (1974a, b) and Bell and Nelkin $(1977,1978)$;

$$
\begin{aligned}
\frac{d u_{j}}{d t}= & a_{1} k_{j}\left(u_{j-1}^{2}-2 u_{j} u_{j+1}\right)+a_{2} k_{j}\left(u_{j-1} u_{j}-2 u_{j+1}^{2}\right) \\
& -v k_{j}^{2} u_{j}+F_{j}(t)
\end{aligned}
$$

where $k_{j}=2^{j} k_{0}$ stands for a discretized scalar wavenumber band (shell), $2^{j-1 / 2} k_{0}<|k|<2^{j+1 / 2} k_{0}$, which real velocity variables $u_{j}$ are associated with, and $a_{1}, a_{2}$ are respectively positive and negative constants. The energy spectrum is then defined as $E\left(k_{j}\right)=u_{j}^{2} / 2$. Desnyansky and Novikov, and Bell and Nelkin were interested in the intermittency of turbulence, and studied in detail both the steady and the time-dependent solutions of this cascade model, finding a relation between the ratio of the backward to the forward energy cascades and an intermittency exponent appearing in the energy spectrum. We remark that dependent variables in most of these models were interpreted as some statistical quantities like the energy spectrum, and the intermittency were discussed with non-chaotic solutions. A similar cascade model was also proposed by Gledzer (1973) for the 2-D Navier-Stokes turbulence. The velocity variables are real in the model, and he obtained two kinds of solutions with $k^{-3}$-spectrum in the forward enstrophy cascading range, and with $k^{-5 / 3}$-spectrum in the backward energy cascading range, respectively.

Later in 1980s, Gloaguen et al. (1985) and Grappin et al. (1986) studied a different type of cascade model of MHD turbulence, in which the dependent variables representing the velocity and magnetic fields behave in a chaotic manner with the time-averaged energy spectrum obeying the Kolmogorov scaling law. In this chaotic cascade model they numerically obtained the Lyapunov spectrum and verified that the Kaplan-Yorke dimension of the underlying strange attractor is consistent with the Kolmogorov scaling law.

A chaotic cascade model, corresponding to the 2-D homogeneous isotropic Navier-Stokes turbulence, was studied by Yamada and Ohkitani (1988). They showed that there are chaotic solutions in which the time-averaged energy spectrum has the $k^{-3}$ form in the enstrophy cascading range, in Gledzer's cascade model with a modification of the forcing 
and dissipation terms. They obtained the Lyapunov spectrum of its chaotic attractor, and showed that the energy spectrum obeys the Batchelor-Kraichnan-Leith (BKL) scaling law of 2-D Navier-Stokes turbulence, and that there are many Lyapunov exponents $\lambda$ close to zero, resulting in a divergence of the density distribution function $f(\lambda)$ of the Lyapunov exponents at $\lambda=0$.

A chaotic cascade model for 3-D homogeneous isotropic Navier-Stokes turbulence was then proposed by Yamada and Ohkitani (1987), who modified the Gledzer model by complexifying the dependent variables and requiring the energy conservation. They did this modification because preliminary computation showed a statistically unstable behavior of the solution when the dependent variables was kept real. Another reason for this modification is that the model is expected to have a similar form to the Fourier transformed Navier-Stokes equation, which has complex velocity variables with effectively two degrees of freedom for each wavenumber because of the incompressible condition for fluid. The complexified model also has two degrees of freedom for each wavenumber shell, in contrast with the original Gledzer model which has one degree of freedom for each wavenumber. Hereafter we focus our attention to this complexified Gledzer's model, which is often called GOY (Gledzer-Ohkitani-Yamada) model.

This shell model is constructed in a discrete wavenumber space, defined as $k_{j}=k_{0} q^{j}(q=2,1 \leq j \leq N)$. The dependent variable $u_{j}$, which is associated with the wavenumber $k_{j}$, is considered to stand for the velocity component whose wavenumber $k$ lies in the wavenumber shell $k_{j}<|k|<k_{j+1}$. We then define the innerproduct of velocities $\left\{u_{j}=u_{j}^{R}+i u_{j}^{I}\right\}$ and $\left\{v_{j}=v_{j}^{R}+i v_{j}^{I}\right\}(1 \leq j \leq N)$ as $\sum_{j=1}^{N}\left(u_{j}^{R} v_{j}^{R}+u_{j}^{I} v_{j}^{I}\right)$ and the superscripts $R$ and $I$ denote the real and the imaginary parts, respectively. Note that the phase space of the system is a real $2 N$-dimensional space, and the inner product is not the Hermitian one. The energy $E_{I}$, the enstrophy $Q_{I}$ and the energy spectrum $E_{I}(k)$ at an instance are then defined respectively as $E_{I}=\sum_{j=1}^{N}\left|u_{j}\right|^{2} / 2, Q_{I}=\sum_{j=1}^{N} k_{j}^{2}\left|u_{j}\right|^{2} / 2, E_{I}\left(k_{j}\right)$ $=\left|u_{j}\right|^{2} /\left(2 k_{j}\right)$.

We assume that each evolution equation for $u_{j}$ is quadratically nonlinear, and consists of nonlinear interactions among $u_{j-2}, u_{j-1}, u_{j}, u_{j+1}, u_{j+2}$. The conservation of the phase volume, $\sum_{j=1}^{N}\left(\partial \dot{u}_{j}^{R} / \partial u_{j}^{R}+\partial \dot{u}_{j}^{I} / \partial u_{j}^{I}\right)=0$, is also assumed to hold in the inviscid case, where the dot denotes the time derivative. These requirements do not determine the form of the shell model uniquely, of course. Remembering that our aim is to see what kind of chaotic attractor can be related to the scaling law of the Kolmogorov type, we adopt the following simple set of equations,

$$
\begin{aligned}
& \left(\frac{d u_{j}}{d t}+v k_{j}^{2}\right) u_{j} \\
= & i\left(c_{j}^{(1)} u_{j+2}^{*} u_{j+1}^{*}+c_{j}^{(2)} u_{j+1}^{*} u_{j-1}^{*}+c_{j}^{(3)} u_{j-1}^{*} u_{j+1}^{*}\right) \\
& +f \delta_{j, l}, \quad(1 \leq j \leq N)
\end{aligned}
$$

where $*$ denotes the complex conjugate, $f$ is a time-independent force, $v$ the kinematic viscosity, $\delta_{j, l}$ Kronecker's delta $(l \in \mathbf{N})$, and $t$ is time. The real constants $c_{j}^{(1)}, c_{j}^{(2)}, c_{j}^{(3)} \quad(1 \leq j \leq N)$ are given as $c_{j}^{(1)}=k_{j}, c_{j}^{(2)}=-\delta k_{j-1}, c_{j}^{(3)}=(\delta-1) k_{j-2} \quad$ except $\quad$ for $c_{1}^{(2)}=c_{1}^{(3)}=c_{2}^{(3)}=c_{N-1}^{(1)}=c_{N}^{(1)}=c_{N}^{(2)}=0$, where $\delta$ is a real parameter. These nonlinear terms conserve the energy $E$. We note that if $\delta$ is larger than unity, the nonlinear terms conserve $E_{I}^{(\alpha)}=\sum_{j=1}^{N} k_{j}^{\alpha}\left|u_{j}\right|^{2}$ together with the energy $E_{I}$, where $\alpha$ is given by $\delta=1+1 / q^{\alpha}$. This property was made use of to study the relation between the cascade process and conserved quantities. We now choose the value of $\delta$ to be $1 / 2$, where we have another inviscid conserved quantity $H_{I}=\sum_{j=1}^{N}(-1)^{j} k_{j}\left|u_{j}\right|^{2}$. This quantity is considered to correspond to the helicity, which is also conserved in the Navier-Stokes equations without viscosity. The number of $N$ is usually taken to be $10-30$, with the forcing term $f \sim 10^{-3}-10^{-5}$ and the viscosity $v \sim 10^{-3}-10^{-7}$.

Numerical calculation of this shell model shows that unsteady solutions are obtained from arbitrary chosen initial conditions with smooth and not too large energy spectra. The unsteady solution is found to be a chaotic solution in the sense that it is a bounded solution with at least one positive Lyapunov number. Long time average of a dynamical quantity is observed to converge to a constant value, suggesting that the motion is governed by an attractor with an invariant measure in phase space. Especially the time average of the energy spectrum $E(k)=<E_{I}(k)>$ shows a $k^{-5 / 3}$ spectral form of Kolmogorov (Fig. 1), which can be observed over about 10 decades of wavenumber in the case of smaller viscosity (Yamada and Ohkitani, 1987). We note that the slope $-5 / 3$ is also observed in real turbulence but over about 2 or 3 decades usually. Moreover, the time average of the energy spectrum is found to obey the Kolmogorov scaling law in which the time average of the energy dissipation rate $\epsilon=2 v\left\langle Q_{I}\right\rangle$ and the viscosity $v$ are the only parameters determining the spectral form, where the bracket denotes the time average (Yamada and Ohkitani, 1987). The energy spectrum is then expressed as

$$
E(k)=\epsilon^{1 / 4} v^{5 / 4} E_{e}\left(k / k_{d}\right)
$$

where $E_{e}$ is a non-dimensional function and $k_{d}=\epsilon^{1 / 4} v^{-3 / 4}$ is the Kolmogorov dissipation wavenumber. Actually in the inertial range, where the energy spectrum $E(k)$ has the Kolmogorov scaling form, the energy flux is fairly constant and equal to the energy dissipation rate (Yamada and Ohkitani, 1989). Thus the K41 scaling law holds for the energy spectrum in the shell model.

The original K41 theory is not restricted to the energy spectrum but describes scaling properties of higher-order moments of velocity. However, it is now well-known that for higher-order quantities the K41 theory, employing only the energy dissipation rate $\epsilon$ and the viscosity $v$ as governing parameters, fails to give the proper scaling exponents of 


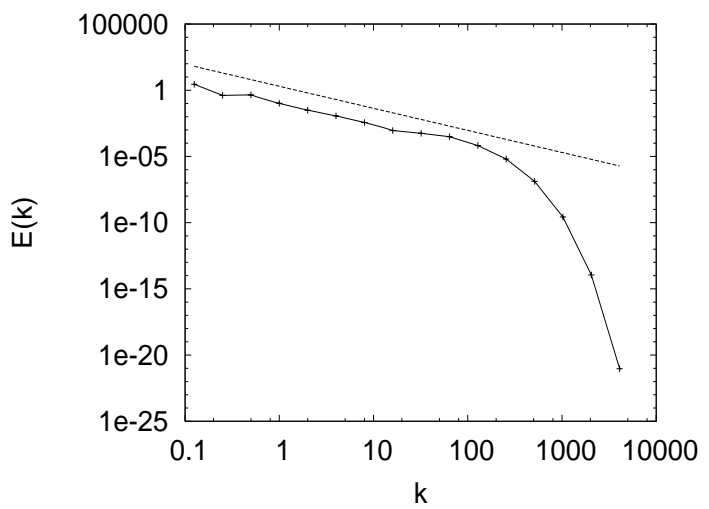

Fig. 1. The time averaged energy spectrum at $v=10^{-4}$. The straight line shows the slope of $-5 / 3$. $f=0.005(1+i), l=1, N=16$.

the structure function of velocity, with the disagreements being called intermittency. It is remarkable that in the shell model the velocity shows a similar deviation of the scaling exponents from K41 theory, and thus has an intermittency property as observed in real fluid turbulence. The intermittency in the shell model has therefore been studied extensively (Jensen et al., 1991: Pisarenko et al., 1993; Yamada et al., 1993; Biferale et al., 1994, 1995; Kadanoff et al., 1995, 1997; Biferale, 2003). We will discuss the intermittency from a viewpoint of UPOs later. Also we note that the effect of the helicitylike invariant and the stability problem of steady solutions have been investigated in the shell model (Kadanoff et al., 1997; Biferale et al., 1998).

\section{Lyapunov spectrum}

Numerical simulation shows that solution orbits of the shell model are chaotic in the phase space. The Lyapunov spectrum is often employed to characterize the chaotic properties of motion. We rewrite the model equation in the following form.

$$
\frac{d \mathbf{u}}{d t}=\mathbf{N}(\mathbf{u})
$$

where

$$
\begin{aligned}
\mathbf{u} & =\mathbf{u}(t) \\
& =\left(u_{1}^{R}(t), u_{1}^{I}(t), u_{2}^{R}(t), u_{2}^{I}(t), \cdots, u_{N}^{R}(t), u_{N}^{I}(t)\right)
\end{aligned}
$$

and $\mathbf{N}$ denotes the sum of the forcing term, the dissipation term and the linear terms. Together with the time evolution of $\mathbf{u}(t)$, we consider the time evolution of infinitesimal displacement $\delta \mathbf{u}(t)$ of the solution $\mathbf{u}(t)$. The displacement $\delta \mathbf{u}(t)$ then obeys the linearized equation of motion around $\mathbf{u}(t)$,

$$
\frac{d \delta \mathbf{u}}{d t}=D \mathbf{N}(\mathbf{u}) \delta \mathbf{u}(t)
$$

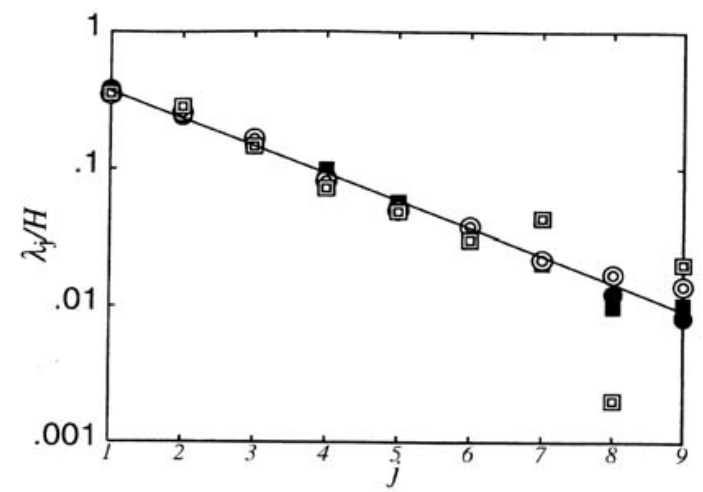

Fig. 2. The Lyapunov exponents $\lambda_{j} / H$ : circles $(N=19)$, squares $(N=22)$, closed squares $(N=24)$, and closed circles $(N=27)$. The dashed line denotes the theoretical prediction: $\lambda_{j} / H=\left(2^{2 / 3}-1\right) 2^{-2 j / 3}$. Reprinted figure with permission from Yamada and Ohkitani (1998, http://link.aps.org/abstract/PRE/v57/ p6257) in Phys. Rev., E57, 6257. Copyright (1998) by the American Physical Society.

where $D \mathbf{N}(\mathbf{u})$ denotes the linearized operator (Fréchet derivative) of $\mathbf{N}(\mathbf{u})$, operating to $\delta \mathbf{u}(t)$. The total dimension of the phase space is $2 N$, and thus we can take linearly independent $2 N$ displacement vectors $\delta \mathbf{u}_{p}(t)(1 \leq p \leq 2 N)$. Then the Lyapunov spectrum is a set of the Lyapunov exponents $\lambda_{1}, \lambda_{2}, \cdots, \lambda_{2 N}$ which are defined by

$$
\begin{aligned}
& \lambda_{1}+\lambda_{2}+\cdots+\lambda_{q} \\
& =\lim _{t \rightarrow \infty} \frac{\left\|\delta \mathbf{u}_{1}(t) \wedge \delta \mathbf{u}_{2}(t) \wedge \cdots \wedge \delta \mathbf{u}_{q}(t)\right\|}{\left\|\delta \mathbf{u}_{1}(0) \wedge \delta \mathbf{u}_{2}(0) \wedge \cdots \wedge \delta \mathbf{u}_{q}(0)\right\|}
\end{aligned}
$$

for $1 \leq q \leq 2 N$ and for almost all initial conditions $\delta \mathbf{u}_{1}(0), \delta \mathbf{u}_{2}(0), \cdots, \delta \mathbf{u}_{q}(0)$. We compute these Lyapunov exponents by a classical method of Shimada and Nagashima(1979) using Gram-Schmidt orthogonalization. Note that these Lyapunov exponents are ordered as $\lambda_{q} \geq \lambda_{q+1}$, and also that some of important chaos parameters are expressed by the Lyapunov exponents. For example, the Lyapunov (KaplanYorke) dimension $D$ of the chaos attractor is given by $D=p+\sum_{j=1}^{p} \lambda_{j} /\left|\lambda_{p+1}\right|, p=\max \left\{m \mid \sum_{j=1}^{m} \lambda_{j} \geq 0\right\}$, and the Kolmogorov-Sinai (KS) entropy $H$ is given by $H=\sum_{j=1}^{q} \lambda_{j}\left(\lambda_{q}>0, \lambda_{q+1} \leq 0\right) .{ }^{1}$

We numerically calculate the Lyapunov exponents of the model with $f=0.005(1+i)$ and $l=4$, some of which are shown in Fig. 2, where the Lyapunov exponents are normalized by the KS entropy $H$, for several values of the viscosity. As the viscosity decreases, the number of the Lyapunov exponents nearly equal to zero increases while the number of large positive Lyapunov exponents remains the

\footnotetext{
${ }^{1}$ Rigorously speaking, $H$ gives only an upper bound of the KS entropy, but the difference is often small and is not discussed in this paper.
} 
same, suggesting that there is an asymptotic expression of the Lyapunov exponents in the limit of $v \rightarrow 0$. Actually the asymptotic expression is obtained by examining supports of the Lyapunov vectors as follows.

We show the support of the Lyapunov vectors in Fig. 3, plotting the squared components of the Lyapunov vectors in its time average, $E^{j}\left(k_{n}\right)=<\left|\delta u_{n}^{j}\right|^{2}>$, where $\delta u_{n}^{j}$ denotes the $n$-th Fourier component of the $j$-th Lyapunov vector, the bracket the time average and each Lyapunov vector is normalized as $\sum_{n}\left|\delta u_{n}^{j}\right|^{2}=1$ for each $j$.

We can see that each Lyapunov vector has a localized support in wavenumber space. The center of the support lies around at $n \sim D / 2$ for the largest Lyapunov exponent $(j=1)$, while it decreases to $n \sim 0$ as $j$ increases to $D / 2$. For $j>D / 2$, the Lyapunov exponents are negative and the central wavenumber of the support increases to $n \sim D / 2$ at $j \sim D$. For $j>D$, two Lyapunov vectors share the same central wavenumber increasing with $j$, and the Lyapunov exponent asymptotically agrees with the reciprocal of the viscous time scale at the wavenumber.

In summary, for each $n(0 \leq n \leq D / 2)$ there are two Lyapunov vectors corresponding to positive and negative Lyapunov exponents, in harmony with the fact that there are two degrees of freedom at each wavenumber in the shell model. For $n \geq D / 2$, on the other hand, the wavenumber components are considered to lie outside the attractor, obeying the simple dissipation dynamics. The suffix of the wavenumber $n \leq D / 2$ is, therefore, considered to correspond to the inertial subrange in the shell model.

This observation leads us to introduce the following set of hypotheses regarding the Lyapunov vectors in the inertial subrange $j \leq D$ for $D \gg 1$.

1. Lyapunov exponents are positive for $1 \leq j \leq D / 2$, and negative for $j>D / 2$.

2. Each Lyapunov vector in wavenumber space is localized around a wavenumber. Let $k_{n_{j}}=k_{0} 2^{n_{j}}$ be the localized wavenumber for $j$-th Lyapunov vector, then $n_{j}$ is given by $n_{j}=D / 2-j+1$ for $1 \leq j \leq D / 2$, and $n_{j}=j-D / 2$ for $D / 2 \leq j \leq D$.

3. In the inertial subrange, the $j$-th Lyapunov exponents $(j \leq D)$ is inversely proportional to the Kolmogorov time scale $\epsilon^{-1 / 3} k_{n_{j}}^{-2 / 3}$.

The last hypothesis is a combination of the Kolmogorov's dimensional argument and the localization of the Lyapunov vectors in wavenumber space. This hypothesis is equivalent to the assumption that the Lyapunov exponent can be represented in terms of the energy dissipation rate $\epsilon$ and the wavenumber $k$ at which the corresponding Lyapunov vector localizes. It should be stressed that the localization of the Lyapunov vectors is thus fundamental to bridge between the chaos dynamical description and the traditional Kolmogorov picture of turbulence.

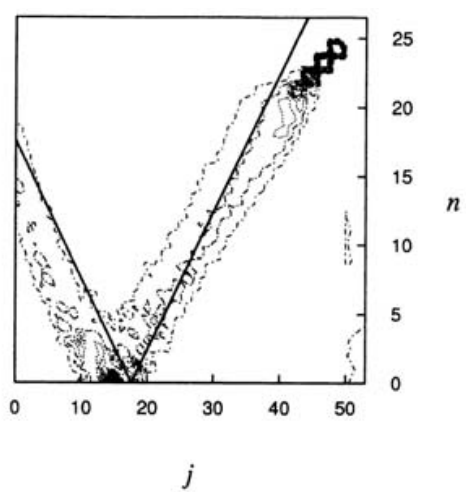

Fig. 3. Time average of squared components of the Lyapunov vectors $\left\langle\left|v_{n}^{j}\right|>(N=27, D=35.0)\right.$ : Contour levels are $0.0489 i(i=1, \cdots, 10)$. Two straight lines represent $n_{j}$ assumed in the hypothesis 2. Reprinted figure with permission from Yamada and Ohkitani (1998, http://link.aps.org/abstract/PRE/v57/p6257) in Phys. Rev., E57, 6257. Copyright (1998) by the American Physical Society.

From the hypotheses above, we obtain the following formulas for the Lyapunov exponents,

$\lambda_{j} \sim\left\{\begin{aligned} \epsilon^{1 / 3} k_{n_{j}}^{2 / 3}=\epsilon^{1 / 3}\left(2 k_{0}\right)^{2 / 3} 2^{(D-2 j) / 3} & \text { for } 1 \leq j \leq D / 2, \\ -\epsilon^{1 / 3} k_{n_{j}}^{2 / 3}=-\epsilon^{1 / 3}\left(2 k_{0}\right)^{2 / 3} 2^{(2 j-D) / 3} & \text { for } D / 2+1 \leq j \leq D .\end{aligned}\right.$

We now normalize the Lyapunov exponents by the KS entropy $H$ as $\lambda_{j} / H$ to eliminate an arbitrary constant in the dimensional analysis. The KS entropy is obtained as

$H=\sum_{j=1}^{D / 2} \lambda_{j} \sim \epsilon^{1 / 3}\left(2 k_{0}\right)^{2 / 3} \frac{2^{D / 3}-1}{2^{2 / 3}-1}$,

and the normalized Lyapunov exponents become

$\frac{\lambda_{j}}{H}= \begin{cases}\frac{\left(2^{2 / 3}-1\right) 2^{2(D / 2-j) / 3}}{2^{D / 3}-1} & \text { for } 1 \leq j \leq D / 2 \\ -\frac{\left(2^{2 / 3}-1\right) 2^{2(j-D / 2) / 3}}{2^{D / 3-1}} & \text { for } D / 2+1 \leq j \leq D\end{cases}$

For $D \gg 1$, the first expression of Eq. (10) reduces to

$\frac{\lambda_{j}}{H}=\left(2^{2 / 3}-1\right) 2^{-2 j / 3}$,

which is depicted in Fig. 2 with a solid line. We can see that the theoretical values and the numerical results agree well and a better agreement is obtained for larger attractor dimension $D$.

It should be remarked here that if the idea of localization of the Lyapunov vectors in the wavenumber space is applicable to the Navier-Stokes turbulence with high Reynolds 
number, a power law is obtained for the density distribution function of the Lyapunov exponents. The localization implies that the Lyapunov exponents are estimated as the reciprocal of the relevant Kolmogorov time scale $\lambda \sim \epsilon^{1 / 3} k^{2 / 3}$, where $k$ is the localization wavenumber of the Lyapunov vector. The number of Lyapunov exponents lying in $[\lambda, \lambda+d \lambda]$ is then proportional to $4 \pi k^{2} d k$, and thus the density distribution function of the Lyapunov exponents $P(\lambda)$ is given by $P(\lambda) d \lambda \sim 4 \pi k^{2} d k$, yielding

$P(\lambda) \sim \lambda^{7 / 2}$.

This power law is, however, beyond the reach of the present ability of numerical computation and its validity is still an open problem, while Eq. (12) is consistent with results of Wang and Gaspart (1992) for KS-entropy and Aurell et al. (1996) for the maximum Lyapunov exponent.

\section{Unstable periodic orbits (UPOs)}

Infinite number of unstable periodic orbits (UPOs) are embedded in a chaotic attractor, and play important roles in chaotic systems (Cvitanović et al., 2005). However, it is usually difficult to find numerically UPOs from chaotic dynamical systems, because UPOs cannot be found by the forward time integration (or iteration) of dynamical systems. So, there are few studies on UPOs of high dimensional dynamical systems. Here we focus our attention to UPOs of GOY shell model with turbulent but not fully developed turbulent regime and discuss relations between UPOs and chaotic (turbulent) solutions. In this section, the external force $f=0.005(1+i)$ is put into the first mode $(l=1)$.

It should be noted here for later convenience that the GOY shell model has a "translational invariance"; if $\mathbf{u}(t)=\left\{u_{j}(t)\right\}$ is a (steady or unsteady) solution of the GOY shell model with the external force $f$, then $R_{\phi} \mathbf{u}(t)$ is also a solution, where $R_{\phi} \mathbf{u}=u_{j}(j \equiv 1), e^{i \phi} u_{j}(j \equiv 2), e^{-i \phi} u_{j}(j \equiv 0)$, with the congruence being defined under $\bmod 3$, and $\phi$ is a continuous real parameter. ${ }^{2}$ The $R_{\phi}$ is called translational transformation because it is similar to the real-space translation which rotates the phases of each Fourier component. We can therefore generate a continuously infinite number of solutions by applying $R_{\phi}$ to a single solution $\mathbf{u}$.

\subsection{Fixed point solutions}

There are some steady solutions for the GOY shell model with the external force $f$ at the first mode. These solutions are captured numerically by the Newton-Raphson method. Hereafter we call these solutions fixed point solutions. While some of them lie outside the chaotic attractor, there is a fixed

\footnotetext{
${ }^{2}$ This is a special case $(\theta=0)$ of the transformation $R_{\phi}^{\theta} \mathbf{u}=e^{i \theta} u_{j}(j \equiv 1), e^{i(\phi-\theta)} u_{j}(j \equiv 2), e^{-i \phi} u_{j}(j \equiv 0) \quad$ which is also a solution of the GOY model without forcing term (Gat et al., 1995).
}

point solution which lies inside and whose Fourier spectrum is quite close to the Kolmogorov $-5 / 3$ spectrum, and the slope remains almost the same against the decreasing viscosity. Numerical simulations show that in the course of the time development, a nearby solution to the fixed point does not stay in the neighborhood of the fixed point, indicating that the fixed point is unstable.

\subsection{Kolmogorov solutions}

We find periodic solutions with a simple time dependence, which was first reported by Kato and Yamada (2002). Assuming that

$u_{j}=a_{j} e^{i \omega_{j} t}\left(a_{j} \in \mathbf{C}, \omega_{j} \in \mathbf{R}\right)$

where $\omega_{j}=0(j \equiv 1),-\theta(j \equiv 2), \theta(j \equiv 0)$ in which $\theta$ is a real constant. The detection of this type of periodic solutions is reduced to finding $\mathbf{a}=\left\{a_{j}\right\}, \theta$ which satisfy the nonlinear algebraic equations:

$\mathbf{F}(\mathbf{a}, \theta)=\mathbf{0}$,

where

$$
\begin{aligned}
F_{j}(\mathbf{a}, \theta)= & v k_{j}^{2} a_{j}-\left\{i \left[a_{j} k_{j} a_{j+1} a_{j+2}+b_{j} k_{j-1} a_{j-1} a_{j+1}\right.\right. \\
& \left.\left.+c_{j} k_{j-2} a_{j-1} a_{j-2}\right]^{*}+f \delta_{j, 1}\right\} \quad(j \equiv 1) \quad(15) \\
F_{j}(\mathbf{a}, \theta)= & \left(-i \theta+v k_{j}^{2}\right) a_{j} e^{-i \theta t}-\left\{i \left[\left(a_{j} k_{j} a_{j+1} a_{j+2}\right.\right.\right. \\
& \left.\left.+b_{j} k_{j-1} a_{j-1} a_{j+1}+c_{j} k_{j-2} a_{j-1} a_{j-2}\right) e^{i \theta t}\right]^{*} \\
& \left.+f \delta_{j, 1}\right\} \quad(j \equiv 2) \\
F_{j}(\mathbf{a}, \theta)= & \left(i \theta+v k_{j}^{2}\right) a_{j} e^{i \theta t}-\left\{i \left[\left(a_{j} k_{j} a_{j+1} a_{j+2}\right.\right.\right. \\
& \left.\left.+b_{j} k_{j-1} a_{j-1} a_{j+1}+c_{j} k_{j-2} a_{j-1} a_{j-2}\right) e^{-i \theta t}\right]^{*} \\
& \left.+f \delta_{j, 1}\right\} \quad(j \equiv 0) .
\end{aligned}
$$

The zeros of $\mathbf{F}(\mathbf{a}, \theta)=\mathbf{0}$ are obtained numerically also by the Newton-Raphson method. Note that in the case of $\theta=0$, this solution coincides with the fixed point solution. We can numerically find some solutions for $\mathbf{F}(\mathbf{a}, \theta)=0$ with $\theta \neq 0$. These solutions are found unstable in the same manner as in the case of the fixed point solutions. ${ }^{3}$

Figure 4 shows projections of a periodic solution onto the complex plane of $u_{j}$ at $v=0.0001$. The modulus of each $u_{j}$ of this solution is constant, as seen from the assumed form of the solution. When the number of modes $N$ is not large enough, these solutions do not have smooth energy spectra. As the viscosity decreases and $N$ is taken larger, the energy spectra of these solutions is observed to have a longer $-5 / 3$ slope in accordance with the celebrated Kolmogorov spectrum. On this account, we call these the "Kolmogorov solutions". It should be remarked that the Kolmogorov solution

\footnotetext{
${ }^{3}$ Very recently, Olesen and Jensen (2007) found interesting exact periodic solutions of the GOY shell model without dissipation and/or forcing terms. In their solutions the modulus of each complex velocity variable is not constant in contrast with the above Kolmogorov solution.
} 

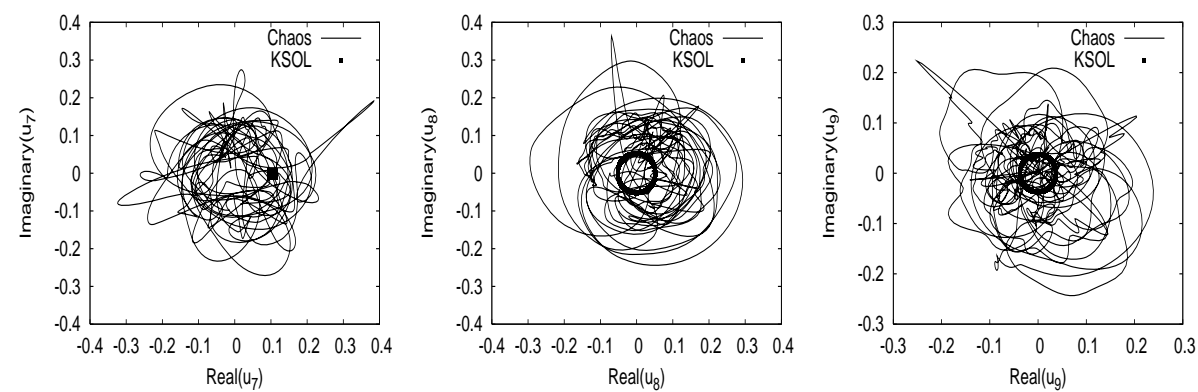

Fig. 4. Behaviors of velocity projected onto each shell ( $j=7$ (left), $j=8$ (middle), $j=9$ (right)) at $N=16, \nu=10^{-4}$. Thin and thick lines represent turbulent solution (Chaos) and Kolmogorov solution (KSOL), respectively.

appears to be embedded in the chaotic attractors which are visualized by an orbit of a chaotic solution in Fig. 4. However, the Kolmogorov solution shares only a small part with the chaotic attractor, although the latter appears to spread around the Kolmogorov solution. Note that the Kolmogorov solution is invariant under the action of $R_{\phi}$.

Figure 5 shows the dependence of the period of the orbit of the Kolmogorov solution upon the viscosity. We can see that as the value of $v$ approaches up to $v_{*} \sim 1.646 \times 10^{-3}$, the period increases rapidly, suggesting that the periodic solution converges to the fixed point solutions which are distributed continuously as obtained under the action of the oneparameter transformation $R_{\phi}$.

There is no spatial structure in GOY shell model, but we can define the $p$ th order structure function $S_{p}$ by $S_{p}\left(k_{j}\right)=<\left|u_{j}\right|^{p}>$, and the scaling exponent $\zeta_{p}$ as to satisfy $S_{p}\left(k_{j}\right)=C k_{j}^{-\zeta_{p}}$ where $C$ is a constant. The deviation of the $p$-th order scaling exponent $\zeta_{p}$ from $p / 3$, the original K41 scaling, has been considered to characterize the intermittent structure of turbulence. In the case of the Kolmogorov solutions, which has the temporally constant modulus $\left|u_{j}\right|$ at each wavenumber $k_{j}$, the scaling exponent $\zeta_{p}$ is found to coincide with $p / 3$, indicating that the Kolmogorov solution has no intermittency. Therefore the Kolmogorov solution realizes the picture of the turbulence which the original theory of K41 predicted.

\subsection{Intermittency solutions}

A different type of periodic solutions from the fixed point and the Kolmogorov solutions was found by Kato and Yamada (2003) in the shell model. The time dependence of these solutions is not so simple as the Kolmogorov ones, and some projections of an orbit are shown in Fig. 6 for $v=0.00195$, $N=12$. This solution is found also by Newton-RaphsonMees method (Mees 1981), but in this case the iteration process has to be performed with the full time evolution in which we have to pay much more attention to stronger instability at higher wavenumber on the orbit than on the orbit of the Kolmogorov solutions.

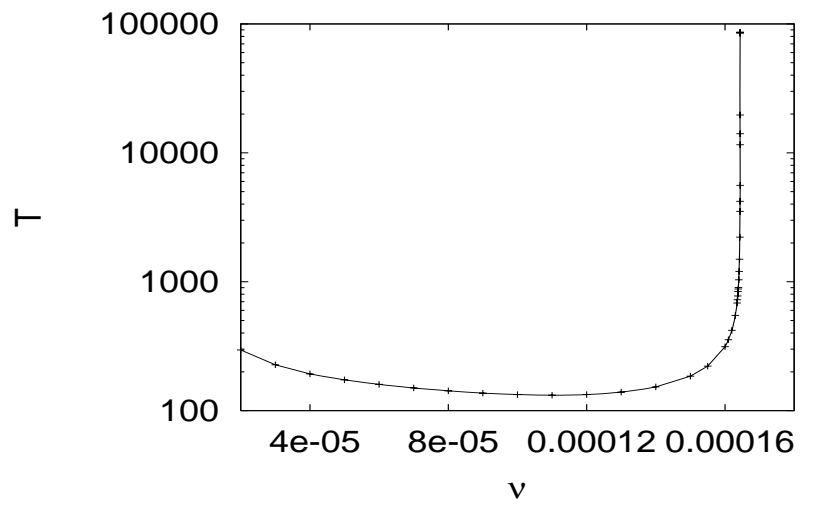

Fig. 5. Period of the Kolmogorov solution at various vs.

The projections of the periodic orbits in Fig. 6 is not circular but has non-uniform and complicated structures, meaning that the behavior of the solution is quite intermittent temporally, which leads us to call the solution the "intermittency solution". The orbit of the intermittency solution $\mathbf{u}_{\text {int }}(t)$ is not invariant to the action of the transformation $R_{\phi}$. The transformed orbit $M \equiv\left\{R_{\phi} \mathbf{u}_{\text {int }}(t) \mid 0 \leq \phi<2 \pi, 0 \leq t<T_{\text {period }}\right\}$ constitutes a two-dimensional manifold in the phase space. We see from Fig. 6 that the intermittency solution appears to be within the chaos attractor and the manifold $M$ mostly covers the chaos attractor. This suggests that properties of the chaos attractor are at least partially reflected by those of the intermittency solution.

We calculate the scaling exponent $\zeta_{p}$ along this intermittency solution (Fig. 7). Unfortunately it is not straightforward to estimate $\zeta_{p}$ numerically because the scaling wavenumber range is not wide enough as $v$ is not sufficiently small and turbulence is not fully developed. Then we employ the "extended self similarity" (ESS) proposed in Benzi et al. (1993) to estimate the values of $\zeta_{p}$, normalizing the structure functions by that of the third order. We show $\zeta_{p}$ thus obtained in Fig. 7, where we find a remarkable coincidence of $\zeta_{p}$ s between the turbulent (chaos) solution and the intermittency solution. 

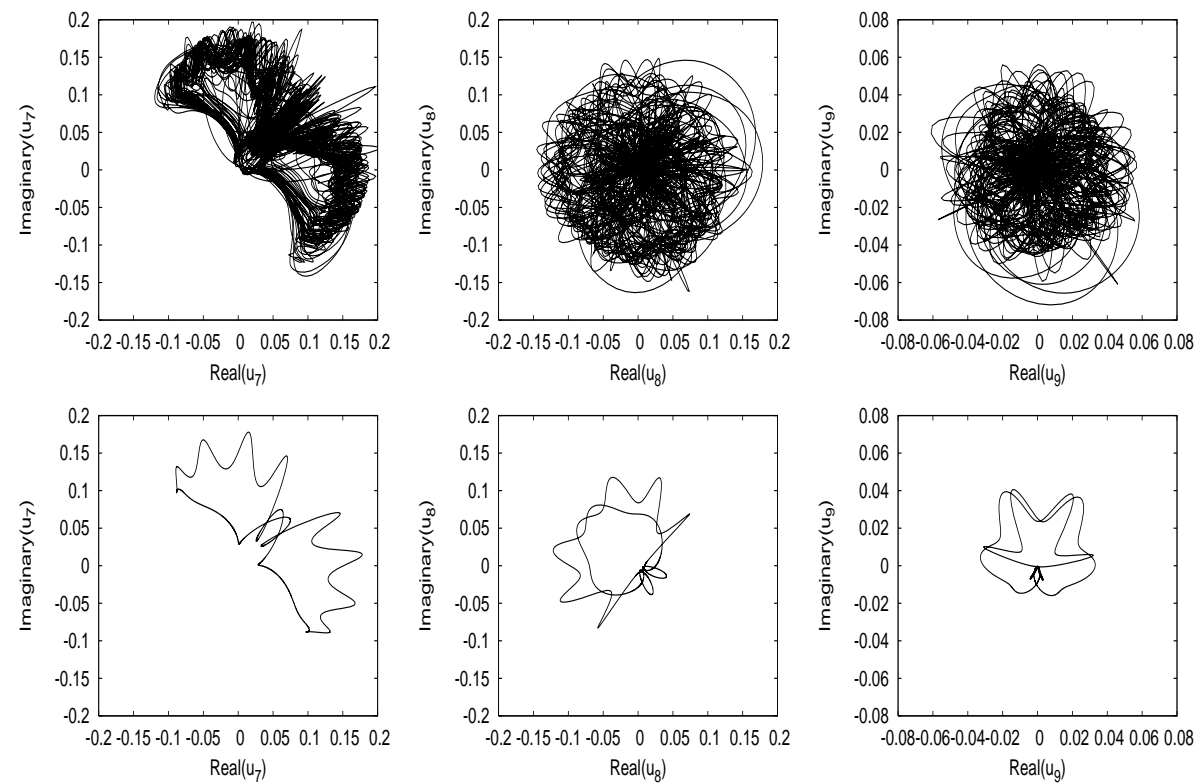

Fig. 6. Behaviors of velocity of turbulent solution (upper) and intermittency solution (lower) projected onto each shell; $j=7$ (left), $j=8$ (middle), $j=9$ (right) at $N=12, v=1.95 \times 10^{-3}$.

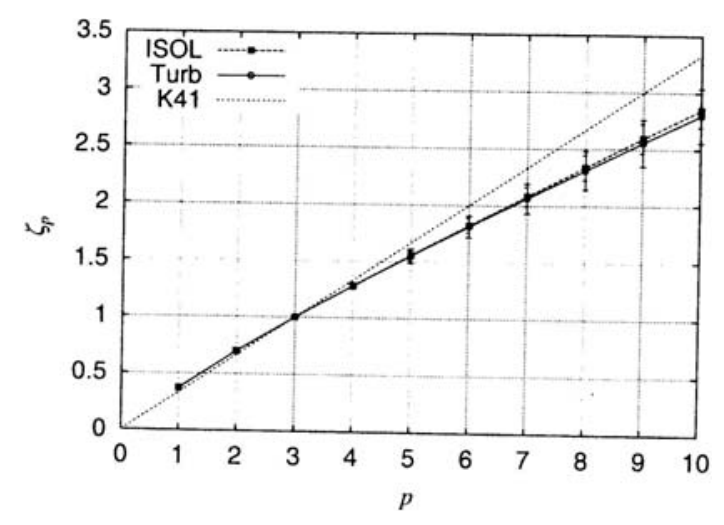

Fig. 7. Scaling exponent $\zeta_{p}$ of the $p$ th order structure function: This is obtained by ESS fitting for the intermittency solution (squares) and turbulent solution (circles). The error bars show the standard deviations obtained from the method of the least squares. The dotted line corresponds to $\mathrm{K} 41\left(\zeta_{p}=p / 3\right)$. Reprinted figure with permission from Kato and Yamada (2002).

This coincidence means that the intermittency solution gives an essential property of the shell model turbulence. In addition, it is observed that the probability distribution function (pdf) of the velocity component $u_{j}$ of the chaos solution is well approximated by that of the intermittency solution, while the Kolmogorov solution fails in the approximation because of its constant modulus of $u_{j}$. It is surprising that only one UPO can give good approximation to the phase space structure and statistical property of the turbulent (chaotic) be- havior. But a further study, which will be reported elsewhere, suggests that this is not so unusual.

Actually there are some studies of fluid turbulence from viewpoints of UPOs in similar contexts. Kawahara and Kida (2001) detected a UPO of Couette flow of Navier-Stokes turbulence, and obtained a remarkable agreement of an averaged velocity profile on the single UPO. The UPO shows a series of typical events of Couette flow as well. Van Veen et al. (2005) detected UPOs of high-symmetry isotropic flow of Navier-Stokes turbulence and found that the energy dissipation rate of one of the UPOs appears to converge to a nonzero value as the Renolds number increases, suggesting that the UPO corresponds to the isotropic turbulence of fluid motion, and Kazantsez (2001) studied UPOs of geometric fluid model. However, it should be noted that in all of these studies Reynolds number is relatively low and the number of detected orbits is small. Many problems on UPOs of fluid and MHD turbulence are left to the future work.

\section{Conclusions}

In this paper we review the shell model of fluid turbulence, which shows statistical properties similar to real fluid turbulence, from the viewpoint of the Lyapunov analysis and unstable periodic orbits. In the shell model, the asymptotic form of the Lyapunov spectrum in the inviscid limit is obtained by Kolmogorov similarity argument using the observation that the corresponding Lyapunov vectors are localized in wavenumber space. Therefore the localization is the key to bridging between the traditional similarity arguments 
of Kolmogorov type and the chaos dynamics of turbulence. This localization discussion is applied to the real 3-D turbulence to yield the density distribution function $P(\lambda)$ of the Lyapunov exponents as $P(\lambda) \sim \lambda^{7 / 2}$.

We also discussed UPOs embedded in the chaotic attractor of the shell model turbulence. We found three kinds of UPOs. One is the fixed point solution corresponding to a steady solution with Kolmogorov spectrum of $k^{-5 / 3}$. The second periodic solution (Kolmogorov solution) has also $k^{-5 / 3}$ spectrum with the modulus of each complex velocity variable being constant while the phase rotates at a constant angular velocity. The scaling exponents of the structure function in Kolmogorov solution obey faithfully the Kolmogorov scaling law, $\zeta_{p}=p / 3$, in contrast with the turbulent solution in which $\zeta_{p}$ deviates from $p / 3$. In the third periodic solution (intermittency solution), the solution orbit is not so simple as the Kolmogorov solutions. The orbit consists of bursting states together with laminar states, the latter of which takes longer time than the former. The energy spectrum of the intermittency solution obeys $k^{-5 / 3}$ law, and the scaling exponents of the structure function show a clear deviation from $p / 3$ as the turbulent solution. Moreover, the scaling exponents $\zeta_{p}$ remarkably coincide with that of the turbulent solution, suggesting that the intermittency solution is a skeleton of the chaotic attractor. It should be remarked that the set of the intermittency solutions generated by a continuous transformation due to a rotational invariance of the equation of motion, almost covers the chaotic attractor in phase space.

It is still an open problem why a single periodic orbit, corresponding to the intermittency solution, can capture the chaotic properties of the chaos solutions. Research on this subject is now on progress and will be reported elsewhere.

Edited by: T. Hada

Reviewed by: two anonymous referees

\section{References}

Aurell, E., Boffeta, G., Crisanti, A., Paladin, G., and Vulpiani, A.: Predictability in systems with many characteristic times: The case of turbulence Phys. Rev. E, 53, 2337-2349, 1996.

Benzi, R., Ruiz, G., Ciliberto, S., and Tripiccianoe, R.: Extended self-similarity in the dissipation range of fully developed turbulence, Europhys. Lett., 24, 275-279, 1993.

Bell, T. L. and Nelkin, M.: Nonlinear cascade models for fullydeveloped turbulence, Phys. Fluids, 20, 345-350, 1977.

Bell, T. L. and Nelkin, M.: Time dependent scaling relations and a cascade model of turbulence, J. Fluid Mech., 88, 369-391, 1978.

Benzi, R., Biferale, L., and Parisi, G.: On intermittency in a cascade model for turbulence, Physica D, 65, 163-171, 1993.

Biferale, L.: Shell models of energy cascade in turbulence, Ann. Rev. Fluid Mech., 35, 441-468, 2003.

Biferale, L., Blank, M., and Frisch, U.: Chaotic Cascades with Kolmogorov 1941 Scaling, J. Statist. Phys., 75, 781-795, 1994.
Biferale, L., Lambert, A., Lima, R., and Paladin, G.: Transition to chaos in a shell model of turbulence, Physica D, 80, 105-119, 1995.

Biferale, L., Pierotti, D., and Toschi, F.: Helicity transfer in turbulent models, Phys. Rev. E, 57, R2515-2518, 1998.

Bohr, T., Jensen, M., Paladin, G., and Vulpiani, A.: Dynamical Systems Approach to Turbulence, Cambridge Nonlinear Science Series, 1998.

Cvitanović, P., Artuso, R., Mainieri, R., Tanner, G., and Vattay, G.: Chaos: Classical and Quantum, ChaosBook.org, Niels Bohr Institute, 2005.

Desnyansky, V. N. and Novikov, E. A.: The evolution of turbulence spectra to the similarity regime, Izvestiya Akademii Nauk SSSR Fizika Atmospery I Okeana, 10, 127-136, 1974a.

Desnyansky, V. N. and Novikov, E. A.: Models of Cascade Process in Turbulent Flows, Prikladnaya Matematika I Mekhanika, 38, 507-513, 1974b.

Frisch, U., Sulem, P. L., and Nelkin, M.: A simple dynamical model of intermittent fully developed. turbulence, J. Fluid Mech., 87, 719-736, 1978.

Gat, O., Procaccia, I., and Zeitak, R.: Breakdown of dynamic scaling and intermittency in a cascade model of turbulence, Phys. Rev. E, 51, 1148-1154, 1995.

Gledzer, E. B.: System of hydrodynamic type admitting two quadratic integrals of motion, Sov. Phys. Dokl., 18, 216-217, 1973.

Grappin, R., Leorat, J., and Pouquet, A.: Computation of the dynamics of a model of fully developed turbulence, J. Phys., 47(7), 1127-1136, 1986.

Gloaguen, C., Leorat, J., Pouquet, A., and Grappin, R.: A scalar model for MHD turbulence, Physica D, 51, 154-182, 1985.

Jensen, M. H., Paladin, D., and Vulpiani, A.: Intermittency in a cascade model for three-dimensional turbulence, Phys. Rev. A, 43, 1991, 798-805.

Kadanoff, L., Lohse, D., and Schörghofer, N.: Scaling and linear response in the GOY turbulence model, Physica D, 100, 165186, 1997.

Kadanoff, L., Lohse, D., Wang, J., and Benzi, R.: Scaling and dissipation in the GOY shell model, Phys. Fluids, 7, 617-629, 1995.

Kato, S. and Yamada, M.: Unstable periodic solutions of a shell model turbulence, RIMS surikaiseki kenkyujyo koukyuroku, 1285, 226-233 (in Japanese), 2002.

Kato, S. and Yamada, M.: Unstable periodic solutions embedded in a shell model turbulence, Phys. Rev. E, 68(2), R25 302-25 305, 2003.

Kawahara, G. and Kida, S.: Periodic motion embedded in plane Couette turbulence: regeneration cycle and burst, J. Fluid Mech., 449, 291-300, 2001.

Kazantsev, E.: Sensitivity of the attractor of the barotropic ocean model to external influences: approach by unstable periodic orbits, Nonlin. Processes Geophys., 8, 301-311, 2001, http://www.nonlin-processes-geophys.net/8/301/2001/.

Kida, S., Yamada, M., and Ohkitani, K.: Route to Chaos in a Navier-Stokes Flow, Lecture Notes in Numerical and Applied Analysis, 10, 31-47, 1989.

Kolmogorov, A. N.: Dissipation of energy in a locally isotropic turbulence, Doklady Akad. Nauk SSSR, 32, 141 (English translation in: American Mathematical Society Translations 1958, Series 2, Vol 8, p. 87, Providence R.I), 1941. 
Kolmogorov, A. N.: A refinement of previous hypotheses concerning the local structure of turbulence in a viscous incompressible fluid at high reynolds number, J. Fluid Mech., 13, 82-85, 1962.

Meneveau, C. and Sreenivasan, K. R.: Simple multifractal cascade model for fully developed turbulence, Phys. Rev. Lett., 59, 1424 1427, 1987.

Olesen, P. and Jensen, M. H.: Exact periodic solutions of shell models of turbulence, arXiv:0705.3123v2, 2007.

Parisi, G. and Frisch, U.: On the singularity structure of fully developed turbulence, in: Turbulence and Predictability in Geophysical Fluid Dynamics, edited by: Ghil, M., Benzi, R., Parisi, G., 84-87, Amsterdam: North-Holland, Park JT, 1985.

Pisarenko, D., Biferale, L., Couvoisier, D., Frisch, U., and Vergassola, M.: Further results on multifractality in shell models, Phys. Fluids A, 5, 2533-2538, 1993.

Shimada, I. and Nagashima, T.: A Numerical Approach to Ergodic Problem of Dissipative Dynamical Systems, Progress of Theoretical Physics, 61, 1605-1616, 1979. van Veen, L., Kida, S., and Kawahara, G.: Periodic motion representing isotropic turbulence, Fluid Dynam. Res., 38(1), 19-46, 2006.

Wang, X.-J. and Gaspard, P.: $\epsilon$ entropy for a time series of thermal turbulence, Phys. Rev. A, 46, R3000-3003, 1992.

Yamada, M. and Ohkitani, K.: Lyapunov spectrum of a chaotic model of three-dimensional turbulence, J. Phys. Soc. Japan, 56, 4210-4213, 1987.

Yamada, M. and Ohkitani, K.: The constant of motion and the inertial subrange spectrum in fully developed model turbulence, Phys. Lett. A, 124, 165-169, 1988.

Yamada, M., Kida, S., and Ohkitani, K.: Unstable and Turbulent Motion of Fluid, edited by: Kida, S., World Scientific, Singapore, 188-199, 1993.

Yamada, M. and Ohkitani, K.: Asymptotic formulas for the Lyapunov spectrum of fully developed shell model turbulence, Phys. Rev. E, 57, R6257-6260, 1998. 